\title{
Identificación genotípica de Staphylococcus con fenotipo meticilino resistente aislados de muestras de humanos, animales y ambiente
}

\author{
Genotype identification of Staphylococcus with methicillin-resistant phenotype \\ isolated from human, animal and environmental samples
Luis José Espejo ${ }^{1}$, Karen Lorena Rodríguez ${ }^{1}$, Martha Fabiola Rodríguez ${ }^{2}$, Arlen Patricia Gómez Ramírez ${ }^{3}$

\section{Resumen}

El objetivo del estudio fue dentificar el gen mecA-1 de aislados de Staphylococcus con fenotipo meticilino resistente (SMR) obtenidos de muestras biológicas y superficies de una clínica veterinaria. Se seleccionaron nueve aislamientos con fenotipo SMR clasificado por el sistema automatizado VITEK. El género y la variabilidad se corroboraron por amplificación y secuenciación de los genes $16 \mathrm{~S}$ rRNA y tuf. También se estableció la presencia del gen mecA-1 por PCR. En los nueve aislamientos con fenotipo SMR se identificaron los genes $16 \mathrm{~S}$ rRNA y tuf, evidenciando un $99 \%$ de similitud con cepas de referencia; sin embargo, solo en cinco muestras se encontró el gen mecA-1. Los resultados demuestran una mayor identificación del genotipo MR en superficies intrahospitalarias, lo que evidencia la necesidad del control y seguimiento de estas cepas en hospitales veterinarios debido a las implicaciones que tienen en salud pública.

Palabras clave: mecA; resistencia antimicrobiana; Staphylococcus spp; hospital veterinario

\section{AbSTRACT}

The aim of this study was to identify the mecA-1 gene of Staphylococcus isolates with methicillin-resistant phenotype (SMR) obtained from biological samples and surfaces of a veterinary clinic. Nine isolates with SMR phenotype classified by the automated

${ }^{1}$ Facultad de Ciencias Agropecuarias, Universidad de La Salle, Colombia

${ }^{2}$ Facultad de Ciencias de La Salud, Universidad de La Salle, Colombia

${ }^{3}$ Facultad de Medicina Veterinaria y de Zootecnia, Universidad Nacional de Colombia, Sede Bogotá, Colombia

${ }^{4}$ E-mail: apgomezr@unal.edu.co

Recibido: 8 de mayo de 2018

Aceptado para publicación: 24 de octubre de 2018 
VITEK system were selected. The genus and the variability were corroborated by amplification and sequencing of the $16 \mathrm{~S}$ rRNA and tuf genes. The presence of the mecA1 gene was also established by PCR. In the nine isolates with SMR phenotype, the $16 \mathrm{~S}$ rRNA and $t u f$ genes were identified, showing $99 \%$ similarity with reference strains; however, only in five isolates was found the $m e c A-1$ gene. The results showed a greater identification of the MR genotype in intra-hospital surfaces, which indicates the need for control and monitoring of these strains in veterinary hospitals due to the implications they have on public health.

Key words: $m e c A$; drug resistance; Staphylococcus spp; veterinary hospital

\section{INTRODUCCIÓN}

El género Staphylococcus comprende más de 30 especies, la mayoría comensales y oportunistas que hacen parte de la microbiota de la piel y las mucosas, con gran capacidad de adaptarse a diversos huéspedes y sobrevivir por meses en superficies inanimadas (Otto, 2010). S. aureus es la especie patógena del género, aunque en los últimos años los estafilococos coagulasa negativos (ECN) han emergido como agentes infecciosos de humanos y animales, debido a la facilidad con la que adquieren genes de resistencia antimicrobiana y de virulencia (Zuniga et al., 2015; Foster, 2017).

El uso inadecuado de antibióticos genera una presión selectiva sobre las cepas resistentes, siendo de especial interés en salud pública Staphylococcus aureus meticilino resistente (SAMR) (WHO, 2014; Van Duin y Paterson, 2016). Existe un número limitado de clones de $S$. aureus y otras especies de Staphylococcus resistentes a la meticilina (SMR) diseminados en el mundo, que pudieron tener su origen a partir de un ancestro común o de varios clones que adquirieron el gen $m e c \mathrm{~A}$, por medio de transferencia horizontal (Ito et al., 2012).

El gen mecA codifica para la variante de la proteína unidora de penicilina (PBP2a) una transpeptidasa de baja afinidad por los betalactámicos que hace que la bacteria puede continuar con el ensamble de la pared en presencia del antibiótico. Existen cuatro genotipos del gen mec clasificados según el grado de homología con el primer gen mecA secuenciado de la cepa de $S$. aureus N315: mecA con una homología mayor al $95 \%$ y dos variantes identificadas (mecA1 y mec $\mathrm{A} 2$ ), mec B aislado de Macrococcus caseolyticus con un $60 \%$ de homología y mecC aislado de S. aureus con $68 \%$ de homología (Ito et al., 2012). Este gen se encuentra en el cassette cromosómico de Staphylococcus (SCC), que corresponde a un elemento móvil (isla genómica) para el intercambio de genes. Hasta el momento se han identificado cinco tipos de SCCmec (I, II, III, IV, V), de acuerdo con la combinación de los complejos ccr y mec (de Lencastre et al., 2009). Los genes ccr (AB1, AB2, AB3, AB4 y C) codifican para las recombinasas, sitios específicos que integran el SCC en el cromosoma y el complejo mec, las cuales incluyen los genes reguladores mecR1 y mecI y las secuencias de inserción (IS) 431 y 1272. Estos genes son los encargados de acoplar y agrupar otros genes de resistencia similares a IS (Hanssen y Ericson Sollid, 2006). S. sciuri se considera el origen de mecA; sin embargo, en todos los casos, el gen parece encontrarse en los ECN, los cuales transportan varios tipos de SCCmec y pueden servir como reservorios, especialmente cuando comparten el mismo nicho donde existe una presión selectiva por el uso de antibióticos (Stapleton y Taylor, 2002; Hanssen y Ericson Sollid, 2006). 
El primer aislamiento de SAMR se realizó en ambientes hospitalarios (SAMR-HA) del Reino Unido en 1961 (MCTC 10442, $\mathrm{SCC}$ ec I), denominado el clon arácnido por su diseminación en todo el mundo. En 1982 se aisló la cepa N315 (SCCmec II) en Japón y posteriormente en Nueva York, y la cepa 85/2082 (Sccmec III) en 1985 en Nueva Zelanda. Estas últimas se distribuyeron en todo el mundo (Deurenberg et al., 2007). En 1990 se reportó un brote de SAMR (SCCmec $\mathrm{IV}$ o $\mathrm{V}$ ) en personas que tenían poco contacto con hospitales o sus trabajadores y, por lo tanto, no presentaban factores de riesgo específicos. Estas cepas fueron denominadas cepas asociadas a la comunidad (SAMR$\mathrm{CA})$, las cuales tienen un espectro más limitado de resistencia, son susceptibles a la mayoría de los $\beta$-lactámicos y poseen la habilidad de diseminarse rápidamente con clones predominantes por varias regiones geográficas (Van Duin y Paterson, 2016). Esta misma característica ha sido reportada en la cepa ST398 de SAMR aislada de cerdos, bovinos, aves de corral, animales de compañía y personas en estrecho contacto con animales, denominadas cepas asociadas a la producción pecuaria (SAMR-LA) (Heikinheimo et al., 2016).

Debido a la importancia clínica de los SMR, es evidente la necesidad de implementar herramientas diagnósticas más sensibles y específicas para la identificación fenotípica y genotípica de las cepas aisladas a partir de muestras obtenidas de ambientes hospitalarios, asociados a la comunidad y al sector agropecuario (Schmidt et al., 2017). Los estudios realizados en veterinaria son limitados comparados con los reportes en medicina humana (Hoet et al., 2011; van Balen et al., 2013), lo cual justifica las investigaciones basadas en la identificación, control y seguimiento de la resistencia antimicrobiana en el sector pecuario. El objetivo de este estudio fue identificar el gen $m e c A-1$ de cepas de Staphylococcus con fenotipo SMR aisladas de muestras biológicas y superficies de una clínica veterinaria.

\section{Materiales y Métodos}

\section{Tipo de Estudio}

La investigación correspondió a un estudio descriptivo, no experimental de tipo transversal. Los aislamientos que se emplearon en este estudio se obtuvieron entre los años 2016 y 2017. Todos los procedimientos fueron revisados y aprobados por el Comité de Ética de la Universidad de La Salle (Bogotá, Colombia).

\section{Aislamientos Bacterianos}

Se incluyeron nueve muestras a partir de las cuales se aislaron cepas de Staphylococcus spp. Tres aislamientos fueron obtenidos de hisopados nasales (dos veterinarios y un estudiante de veterinaria), uno obtenido del cultivo de leche de una vaca con mastitis subclínica y cinco de superficies inanimadas intrahospitalarias en contacto con humanos o animales de una clínica veterinaria universitaria de Bogotá (Colombia). Como controles para las técnicas se utilizaron las cepas ATCC® 43300 de SAMR (positiva al gen mecA) y ATCC $(25923$ SAMS (negativa al gen mecA) (Van Balen, 2013).

Los aislamientos fueron identificados por medio del sistema automatizado Vitek ${ }^{\circledR} 2$ Compact (Biomerieux, Francia). Para este proceso, las cepas fueron cultivadas en medio tripticasa de soya (Difco Laboratories) durante 18 horas antes de la tipificación microbiológica con las tarjetas Vitek ${ }^{\circledR}$ 2- GP (Referencia 21342) de acuerdo con las recomendaciones de los fabricantes. El perfil de susceptibilidad también se determinó mediante Vitek $^{\circledR} 2$ Compact con las tarjetas AST-P577, siguiendo las recomendaciones de la casa comercial. En el análisis se incluyeron las bacterias de fenotipo MR. Adicionalmente la sensibilidad antibiótica se determinó por la prueba estandarizada de difusión en agar Meuller-Hinton o técnica de Kirby-Bauer con sensidiscos de oxacilina $(1 \mu \mathrm{g})$, vancomicina 
$(30 \mu \mathrm{g})$, cefoxitina $(30 \mu \mathrm{g})$, ampicilina $(10 \mu \mathrm{g})$, ciprofloxacina $(5 \mu \mathrm{g})$, eritromicina $(15 \mu \mathrm{g})$, clindamicina $(30 \mu \mathrm{g})$, tetraciclina $(30 \mu \mathrm{g})$, trimetoprim/sulfametoxazol $(25 \mu \mathrm{g})$. El análisis de los perfiles de susceptibilidad antimicrobiana fue realizado de acuerdo con las recomendaciones del Instituto de Normas Clínicas y de Laboratorio - CLSI (CLSI, 2016).

\section{Pruebas Moleculares}

La extracción de ADN se realizó con el kit comercial PureLink ${ }^{\mathrm{TM}}$ Genomic DNA Mini Kit (Cat. N ${ }^{\circ}$. K1820-01, Thermo Fisher Scientific), tomando $2 \times 10^{9}$ UFC de la placa de cultivo con un hisopo estéril y resuspendiendo las colonias en $1 \mathrm{ml}$ de agua grado biología molecular. Se centrifugaron a $5000 \mathrm{x}$ g por $10 \mathrm{~min}$. Se descartó el sobrenadante y se agregó nuevamente $1 \mathrm{ml}$ de agua grado biología molecular para un segundo lavado y repetir el mismo protocolo de centrifugación. Del pellet obtenido se realizó la extracción con el kit, siguiendo las recomendaciones de la casa comercial.

La PCR multiplex del gen 16S rRNA y $m e c A-1$ se realizó de acuerdo con los procedimientos reportados previamente (Geha et al., 1994; Hwang et al., 2011). La prueba de PCR se llevó acabo usando cebadores específicos para cada gen de interés, a partir de las secuencias de los genes de 16S rRNA y mecA reportadas por Relman et al. (1990) para 16S rRNA: Cebador X 5'-GGA ATTCAA A[T/G, 1:1]G AAT TGA CGG GGG C correspondiente a los nucleótidos 911 a 930 y cebador Y 5'-CGG GAT CCC AGG CCC GGG AAC GTA TTC AC, correspondiente a los nucleótidos 1371 a 1390 con un tamaño de producto de 479 pares de bases (pb) y para mecA cebador mecAl 5'-GTA GAAATGACT GAA CGT CCG ATA A que corresponde a los nucleótidos 318 a 342 y cebador mecA2 5'-CCA ATT CCA CAT TGT TTC GGT CTAA complementario para los nucleótidos 603 a 627, amplificando un producto de $310 \mathrm{pb}$ (Song et al., 1987; Tesch et al., 1988). Para el procedimiento de am- plificación del $\mathrm{ADN}$ se utilizó una mezcla de $200 \mu \mathrm{M}$ de dNTP, Tris $10 \mathrm{mM}$ (pH 8.5), 50 $\mathrm{mM} \mathrm{KCl}, 1.5 \mu \mathrm{M} \mathrm{MgCl}_{2}, 1 \mu \mathrm{M}$ de cada cebador (mecA y 16S rRNA), en un volumen final de reacción de $50 \mu \mathrm{l}$. Las reacciones de la PCR multiplex se llevaron a cabo con las siguientes condiciones: $94^{\circ} \mathrm{C}$ por 4 min, 30 ciclos de $94{ }^{\circ} \mathrm{C}$ por $45 \mathrm{~s}, 50{ }^{\circ} \mathrm{C}$ por $45 \mathrm{~s}, 72^{\circ} \mathrm{C}$ por $1 \mathrm{~min}$, con una extensión final de $72{ }^{\circ} \mathrm{C}$ por $2 \mathrm{~min}$.

La PCR del gen tuf fue llevada a cabo de acuerdo con los procedimientos descritos por Hwang et al. (2011) usando los cebadores TUF-F (5'-GCCAGTTGAGGACGTATTCT3') y TUF-R (5'-CCATTTCAGTACCTTCTGGTAA-3'), que amplifican un producto de $412 \mathrm{pb}$. Las reacciones de la PCR se realizaron con los siguientes parámetros: $95^{\circ} \mathrm{C}$ por $15 \mathrm{~min}, 35$ ciclos de $95^{\circ} \mathrm{C}$ por $30 \mathrm{~s}$, $56{ }^{\circ} \mathrm{C}$ por $30 \mathrm{~s}, 72{ }^{\circ} \mathrm{C}$ por $45 \mathrm{~s}$, con una extensión final de $72{ }^{\circ} \mathrm{C}$ por $10 \mathrm{~min}$. Los productos de PCR fueron visualizados en un gel de electroforesis al $2 \%$ teñido con HydraGreen ${ }^{\circledR}$ (ACTGene, NJ, EEUU) y con un transiluminador de luz UV.

Los productos positivos a los genes de interés de la PCR fueron purificados por medio de precipitación en alcohol y secuenciados con BigDye Terminato v3.1 Cycle Sequencing kit (Applied Biosystems, California, EEUU) usando una electroforesis de capilares y un secuenciador ABI3730XL (Applied Biosystems). La edición y el análisis de las secuencias de nucleótidos con la predicción de la secuencia de aminoácidos fue realizada por medio del software MEGA 7 (DNAStar, EEUU). El árbol filogenético fue construido por medio del método de neighbor-joining con el software MEGA 7. En el análisis se incluyeron cuatro secuencias de referencia de Staphylococcus reportadas en el GenBank ( $S$. aureus HM352919.1, S. epidermidis AF298800.1, $S$. haemoliticus HM 032764.1 y S. hominis EU571078.1). Las cepas utilizadas en este estudio como controles también fueron secuenciadas e incluidas en el análisis filogenético. 
Cuadro 1. Identificación, origen y perfil de susceptibilidad por un sistema automatizado y por la técnica de Kirby-Bouer (Vitek ${ }^{\circledR} 2$ / Kirby-Bauer) de aislamientos de Staphylococcus spp obtenidos a partir de muestras biológicas y superficies de una clínica veterinaria. Así mismo, el resultado de PCR de la identificación del gen mecA-1

\begin{tabular}{|c|c|c|c|c|c|c|c|c|c|}
\hline \multirow{3}{*}{ Aislamiento } & \multirow{3}{*}{ Origen } & \multicolumn{7}{|c|}{ Perfil de susceptibilidad Vitek ${ }^{\circledR} 2$ / Kirby-Bauer } & \multirow{3}{*}{ 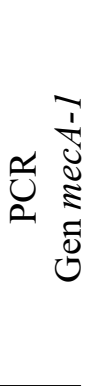 } \\
\hline & & & & 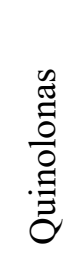 & 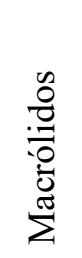 & 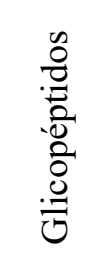 & 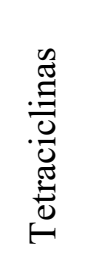 & 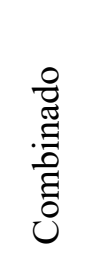 & \\
\hline & & FOX & OX & CIP & E & VAN & $\mathrm{TE}$ & SXT & \\
\hline S. aureus & $\begin{array}{c}\text { Hisopado de } \\
\text { nariz }\end{array}$ & $\mathrm{R} / \mathrm{R}$ & $\mathrm{R} / \mathrm{S}$ & $\mathrm{R} / \mathrm{S}$ & $\mathrm{S} / \mathrm{S}$ & $\mathrm{S} / \mathrm{S}$ & $\mathrm{S} / \mathrm{S}$ & $\mathrm{S} / \mathrm{S}$ & Neg \\
\hline S. epidermidis & $\begin{array}{c}\text { Hisopado de } \\
\text { nariz }\end{array}$ & $\mathrm{R} / \mathrm{R}$ & $\mathrm{R} / \mathrm{S}$ & $\mathrm{S} / \mathrm{S}$ & $\mathrm{R} / \mathrm{S}$ & $\mathrm{S} / \mathrm{S}$ & $\mathrm{R} / \mathrm{S}$ & $\mathrm{S} / \mathrm{S}$ & Neg \\
\hline S. aureus & $\begin{array}{c}\text { Hisopado de } \\
\text { nariz }\end{array}$ & $\mathrm{R} / \mathrm{R}$ & $\mathrm{R} / \mathrm{S}$ & $\mathrm{S} / \mathrm{S}$ & $\mathrm{R} / \mathrm{R}$ & $\mathrm{S} / \mathrm{S}$ & $\mathrm{R} / \mathrm{S}$ & $\mathrm{S} / \mathrm{S}$ & Neg \\
\hline $\begin{array}{l}\text { S. } \\
\text { haemolyticus }\end{array}$ & $\begin{array}{l}\text { Cultivo de } \\
\text { leche }\end{array}$ & $\mathrm{R} / \mathrm{R}$ & $\mathrm{R} / \mathrm{S}$ & $\mathrm{I} / \mathrm{S}$ & $\mathrm{S} / \mathrm{S}$ & $\mathrm{I} / \mathrm{S}$ & $\mathrm{S} / \mathrm{S}$ & $\mathrm{R} / \mathrm{S}$ & Neg \\
\hline S. epidermidis & Superficie & $\mathrm{R} / \mathrm{R}$ & $\mathrm{R} / \mathrm{R}$ & $\mathrm{R} / \mathrm{S}$ & $\mathrm{S} / \mathrm{S}$ & $\mathrm{I} / \mathrm{S}$ & $\mathrm{R} / \mathrm{R}$ & $\mathrm{S} / \mathrm{S}$ & Pos \\
\hline $\begin{array}{l}\text { S. } \\
\text { haemolyticus }\end{array}$ & Superficie & $\mathrm{R} / \mathrm{R}$ & $\mathrm{R} / \mathrm{R}$ & $\mathrm{S} / \mathrm{S}$ & $\mathrm{R} / \mathrm{R}$ & $\mathrm{S} / \mathrm{S}$ & $\mathrm{R} / \mathrm{R}$ & $\mathrm{S} / \mathrm{S}$ & Pos \\
\hline $\begin{array}{l}\text { S. hom } \\
\text { hominis }\end{array}$ & Superficie & $\mathrm{R} / \mathrm{R}$ & $\mathrm{R} / \mathrm{R}$ & $\mathrm{S} / \mathrm{S}$ & $\mathrm{R} / \mathrm{R}$ & $\mathrm{S} / \mathrm{S}$ & $\mathrm{R} / \mathrm{R}$ & $\mathrm{R} / \mathrm{R}$ & Pos \\
\hline $\begin{array}{l}\text { S. hom } \\
\text { hominis }\end{array}$ & Superficie & $\mathrm{R} / \mathrm{R}$ & $\mathrm{R} / \mathrm{R}$ & $\mathrm{S} / \mathrm{S}$ & $\mathrm{R} / \mathrm{R}$ & $\mathrm{S} / \mathrm{S}$ & $\mathrm{S} / \mathrm{S}$ & $\mathrm{S} / \mathrm{S}$ & Pos \\
\hline S. epidermidis & Superficie & $\mathrm{R} / \mathrm{R}$ & $\mathrm{R} / \mathrm{R}$ & $\mathrm{S} / \mathrm{S}$ & $\mathrm{S} / \mathrm{S}$ & $\mathrm{S} / \mathrm{S}$ & $\mathrm{S} / \mathrm{S}$ & $\mathrm{S} / \mathrm{S}$ & Pos \\
\hline S. aureus ${ }^{1}$ & $\begin{array}{c}\mathrm{ATCC}^{\circledR} \\
43300\end{array}$ & $\mathrm{R}$ & $\mathrm{R}$ & $\mathrm{S}$ & $\mathrm{R}$ & $\mathrm{R}$ & $\mathrm{S}$ & $\mathrm{S}$ & Pos \\
\hline S. aureus ${ }^{1}$ & $\begin{array}{c}\mathrm{ATCC}^{\circledR} \\
25923\end{array}$ & $\mathrm{~S}$ & $\mathrm{~S}$ & $\mathrm{~S}$ & $\mathrm{~S}$ & $\mathrm{~S}$ & $\mathrm{~S}$ & S & Neg \\
\hline
\end{tabular}

FOX: cefoxitina, OX: oxacilina, CIP: ciprofloxacina, E: eritromicina, VAN: vancomicina, TE: tetraciclina, SXT: sulfametoxazol.

${ }^{1}$ Corresponden a cepas de referencia $\left(\right.$ ATCC $^{\circ}$ ) de Staphylococcus aureus positivas (43300) y negativas (25923) al gen mecA-1. El perfil de susceptibilidad reportado en el cuadro de estas cepas fue realizado con la técnica de Kirby-Bauer 


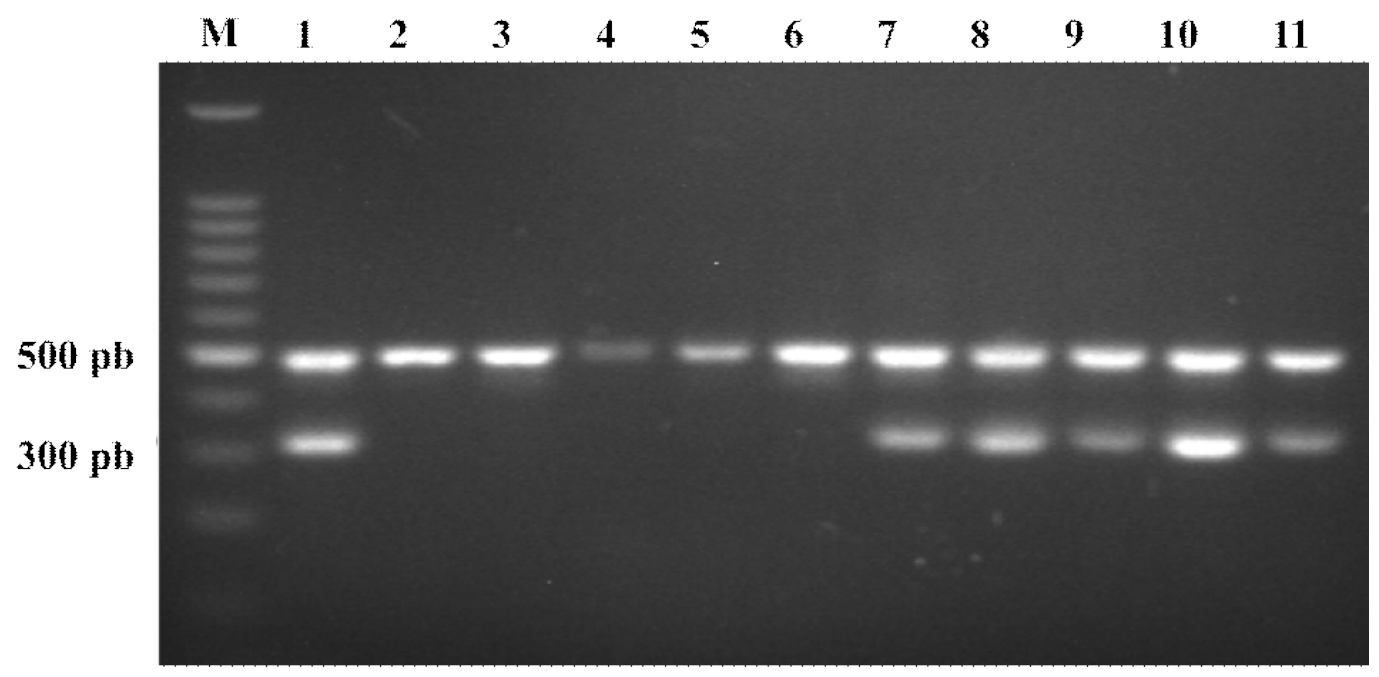

Figura 1.Imagen representativa del ADN de los genes de interés identificados en Staphylococcus spp aislados de muestras biológicas y superficies de una clínica veterinaria. PCR multiplex de los genes $16 \mathrm{~S}$ rRNA (479 pb) y mecA (310 pb). M: marcador de peso molecular. Línea 1: Staphylococcus aureus ATCC $43300^{\circledR}$ (cepa control positivo portador del gen mecA). Línea 2: S. aureus ATCC $25923^{\circledR}$ (cepa control negativo no portador del gen $m e c A$ ). Línea 3: S. aureus aislado de un hisopado de nariz de humano. Línea 4: S. epidermidis aislado de un hisopado de nariz de humano. Línea 5: S. aureus aislado de un hisopado de nariz de humano. Línea 6: S. haemolyticus obtenido de un cultivo de leche de una vaca diagnosticada con mastitis subclínica. Línea 7: $S$. epidermidis aislado de una superficie de una clínica veterinaria. Línea 8: S. haemolyticus aislado de una superficie de una clínica veterinaria. Líneas 9 y 10: S. hom hominis aislados de superficies de una clínica veterinaria. Línea 11: S. epidermidis aislado de una superficie de una clínica veterinaria

\section{Resultados}

\section{Identificación y Perfil de Susceptibilidad Antimicrobiana}

Los nueve aislamientos fueron identificados por el sistema automatizado como $S$. epidermidis (tres cepas), S. aureus (dos cepas), S. haemolyticus (dos cepas) y $S$. hom hominis (dos cepas). En cuanto a los perfiles de susceptibilidad antimicrobiana por Vitek se evidenció que los aislamientos de bacterias obtenidos de muestras biológicas presentaron $57.1 \%$ de resistencia a los antibióticos evaluados frente a $54.3 \%$ de resistencia en las bacterias de las muestras de superficie de la clínica veterinaria. Al analizar los resultados de la técnica de KirbyBauer se detectó $17.9 \%$ de resistencia a los antibióticos evaluados en muestras biológicas en comparación con $48.6 \%$ de resistencia en las bacterias identificadas en muestras de superficie (Cuadro 1), siendo evidente las diferencias en los porcentajes de resistencia a antibióticos obtenidos de las dos técnicas.

La comparación de los resultados generados por el método automatizado y con la técnica de Kirby-Bauer evidenció 100\% de coincidencia en el perfil de resistencia a cefoxitina y $88.9 \%$ para eritromicina y trimetoprim/sulfametoxazol. El porcentaje de coincidencia entre las dos técnicas frente a 


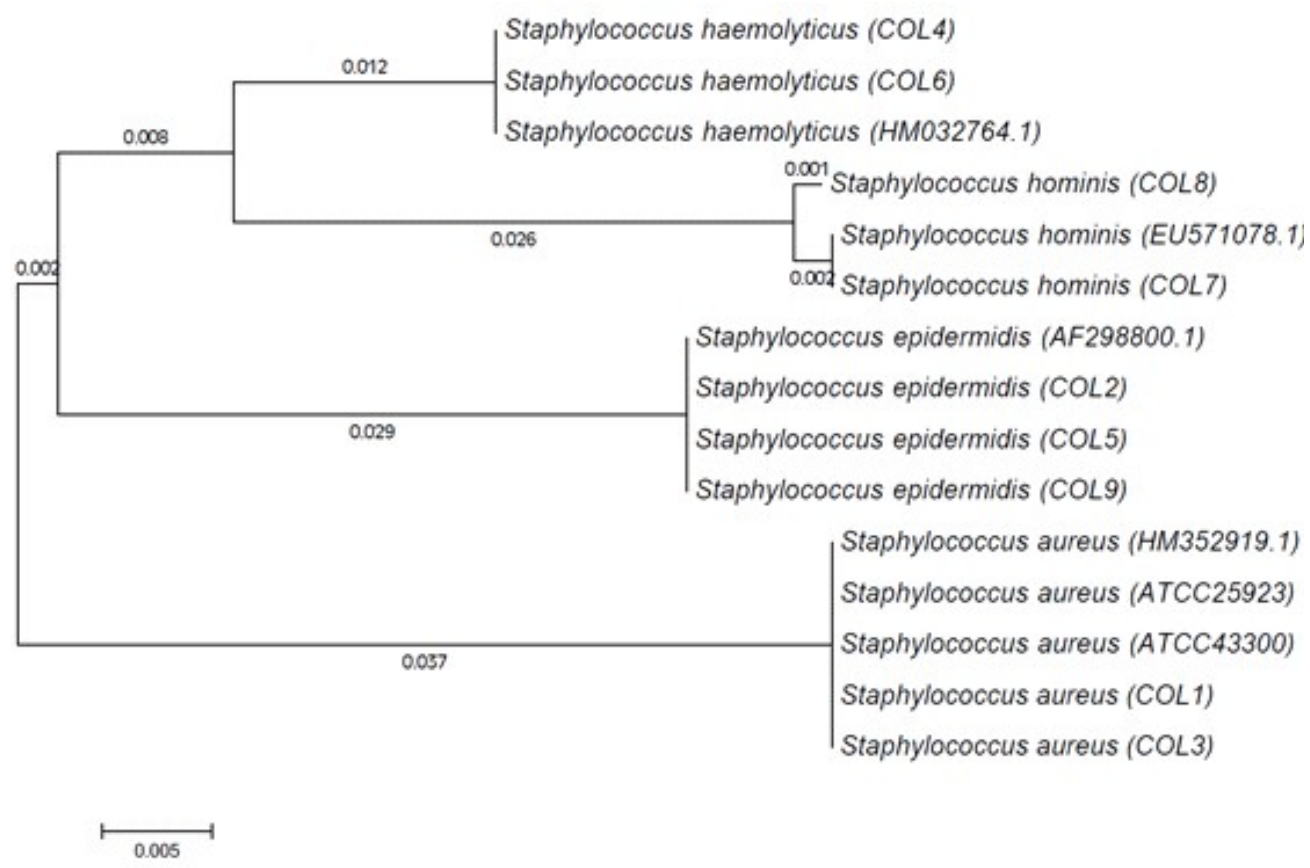

Figura 2.Relación filogenética entre las secuencias de referencia obtenidas del GenBank (Staphylococcus aureus HM352919.1, S. epidermidis AF298800.1, S. haemoliticus HM 032764.1 y $S$. hominis EU571078.1) y los aislamientos obtenidos de muestras biológicas y superficies de una clínica veterinaria. El árbol filogenético fue construido usando el método de neighbor-joining. El largo de cada rama es la diferencia que hay entre cada secuencia de nucleótidos. ATCC 43300: cepa de $S$. aureus positiva al gen mecA-1, ATCC 25923: cepa de $S$. aureus negativa al gen mecA-1, COL1, COL2 y COL3: aislamientos a partir de hisopado de nariz, COL4: aislamiento a partir de cultivo de leche, COL5 a COL9: aislamientos a partir de superficies de una clínica veterinaria. La identificación molecular coincidió con la identificación obtenida por metodologías automatizadas (Vitek)

vancomicina y tetraciclina fue de $77.8 \%$. El menor porcentaje de coincidencia se evidenció para oxacilina y ciprofloxacina (55.6\%).

Todos los aislamientos fueron identificados con fenotipo meticilino resistente por el sistema automatizado; sin embargo, por la técnica de Kirby-Bauer solo los aislamientos a partir de muestras de superficie de la clínica veterinaria (cinco muestras) fueron resistentes tanto a oxacilina como a cefoxitina. Las bacterias obtenidas de muestras biológicas con fenotipo SMR por Vitek presentaron resistencia solo a cefoxitina. Los perfiles de susceptibilidad antimicrobiana evaluados a través de los dos métodos (automatizados y
Kirby-Bauer) también mostraron dos aislamientos de ECN obtenidos de superficies de clínicas veterinarias con fenotipo SMR con resistencia adicional a eritromicina, tetraciclina y trimetoprim/sulfametoxazol (Cuadro 1).

\section{Pruebas Moleculares}

Los nueve aislamientos de Staphylococcus analizados fueron positivos a los genes 16S rRNA y tuf (Figura 1). Después de la secuenciación de los productos de PCR positivos al gen $t u f$ se obtuvieron nueve secuencias con un tamaño promedio de $328 \mathrm{pb}$. El porcentaje de similitud de los aislamientos con las cepas de referencia fue del 99\% (Figura 2). 
Adicionalmente, los cinco aislamientos obtenidos de muestras de superficie fueron positivos al gen mecA (Figura 1). Las cepas positivas al gen mecA coincidieron con el fenotipo SMR por Vitek y Kirby-Bauer. También evidenciaron susceptibilidad a ciprofloxacina, vancomicina y trimetoprim/ sulfametoxazol y resistencia a cefoxitina, oxacilina, eritromicina y tetraciclina con las dos técnicas empleadas. Las especies de Staphylococcus que fueron positivos al gen mecA fueron: S. epidermidis (dos aislamientos), $S$. hom hominis (dos aislamientos) y $S$. haemolyticus (un aislamiento). Las cuatro muestras biológicas con fenotipo SMR por sistema automatizado fueron negativas al gen mecA (Figura 1), lo cual coincide con el perfil de susceptibilidad antibiótica evidenciado por la técnica de Kirby-Bauer.

\section{Discusión}

La resistencia que las bacterias han generado a los antimicrobianos es un problema de salud pública debido a la reducción y limitación de opciones terapéuticas. SMR son bacterias de importancia en medicina humana y veterinaria, ya que ocasionan infecciones nosocomiales, comunitarias y asociadas a la producción pecuaria (Hoet et al., 2011; Van Balen et al., 2013; Rose et al., 2014). De estos SMR, los más estudiados son los clones obtenidos de hospitales humanos, los cuales adicionalmente son multi-drogo resistentes (Van Duin y Paterson, 2016). En esta investigación se logró identificar el gen mecA1 de cepas de Staphylococcus con fenotipo SMR de ambientes hospitalarios veterinarios, de las cuales dos cepas presentaron multirresistencia antibiótica.

En este estudio, el $78 \%$ de las cepas aisladas correspondieron a Estafilococos Coagulasa Negativos (SCN), lo cual está acorde con lo reportado previamente, donde se indica que $S$. epidermidis, $S$. hominis y $S$. haemolyticus son microorganismos que se aíslan de forma común, debido a que perte- necen a la microbiota de la piel y mucosa humana y animal, pero que pueden causar infecciones al comportarse como bacterias oportunistas (Zuniga et al., 2015; Cosseau et al., 2016). S. hominis no es considerado patógeno habitual, pero se ha identificado como patógeno nosocomial multirresistente de las clínicas al igual que $S$. epidermidis, $S$. haemolyticus y $S$. warneri (MendozaOlazarán et al., 2015; Watanabe et al., 2016). Los SCN también están generando un gran interés en salud pública, debido a que presentan mayor resistencia a varias familias de antibióticos en comparación con lo evidenciado con S. aureus (WHO, 2014; Fajardo, 2016; Foster, 2017).

Los resultados de esta investigación evidenciaron diferencias en los patrones de sensibilidad y resistencia antimicrobiana al emplear métodos automatizados en comparación con pruebas convencionales. Los sistemas automatizados permiten una identificación rápida y precisa en comparación con la identificación morfológica o el perfil bioquímico. Sin embargo, la exactitud de estos sistemas puede verse comprometida debido a la expresión variable de características fenotípicas y la naturaleza limitada de las bases de datos, generando en algunos casos resultados ambiguos. Chung et al. (2008) reportaron que en ocasiones estos sistemas presentan restricciones en la identificación del fenotipo SMR en $S$. aureus y otros SCN, debido a las limitaciones en la detección de la expresión heterogénea de resistencia (Aleixandre et al., 2014).

Llama la atención los porcentajes de concordancia de las dos técnicas empleadas para evaluar la sensibilidad antibiótica frente a oxacilina y cefoxitina, siendo menor el porcentaje de concordancia frente a la oxacilina en comparación con la concordancia total frente a cefoxitina. La oxacilina es el antibiótico recomendado por el CLSI para la identificación fenotípica de SMR; sin embargo, se ha demostrado que las técnicas rutinarias que se realizan con este antibiótico no detectan cepas con expresión heterogénea, generan- 
do tanto falsos positivos como negativos $y$, por lo tanto, contribuyendo a la diseminación de cepas resistentes (Safdar et al., 2013). Estudios previos han reportado una sensibilidad del $87.8 \%$ en la identificación de MRSA y una sensibilidad del $73.1 \%$ para las cepas heterorresistentes (Jhon et al., 2009). Por esta razón, los sistemas automatizados han lanzado al mercado tarjetas específicas con cefoxitina, evidenciado un incremento de la sensibilidad y la especificidad de 97.5 y $100 \%$, respectivamente. No obstante, se siguen evidenciando problemas en la identificación del fenotipo SMR en cepas de $S$. epidermis y $S$. hominis hominis (Jhonson et al., 2014).

Se ha demostrado que las cefamicinas (cefoxitina y moxalactam) son potentes inductores de la expresión del gen mecA, directamente relacionado con la mayor síntesis de la proteína PBP2a in vitro (Acosta et al., 2012; Aleixandre et al., 2014). Debido a estos hallazgos, actualmente el CLSI recomienda el uso de cefoxitina en lugar de oxacilina como antibiótico de elección(CLSI, 2016).

La resistencia fenotípica a la oxacilina en cepas de Staphylococcus que carecen del gen mecA, como las cuatro muestras biológicas incluidas en el estudio que presentan una resistencia según el método automatizado a este betalactámico, puede ser debido a la superproducción de $\beta$-lactamasas, especialmente las de clase D denominadas oxacilinasas, que también confieren resistencia a penicilina, cefalosporinas $\mathrm{y}$ carbapenemicos (Mujica et al., 2011). La producción de $\beta$-lactamasas está regulada por dos genes transcritos de forma divergente, blaRl y blaI, cuyos productos génicos tienen homología con aminoácidos de mecRl y mecI, respectivamente (McKineey, 2001). Se ha evidenciado que la transcripción de $m e c A$ y la producción de PBP2a pueden verse afectadas y reguladas por blaI y mecI, lo cual ha sido reportado en especies de SCN como $S$. epidermidis con resistencia a oxacilina (McKineey et al., 2001; Zhang et al., 2001).
En el estudio, gracias a la identificación molecular, se pudo determinar que existen cepas cuya clasificación fenotípica las cataloga como microorganismo MR, pero cuando son puestos a prueba por medio de un diagnóstico molecular se demuestra que puede existir una incoherencia entre la identificación fenotípica y la genotípica de esta condición. Lo anterior soporta la tesis de la implícita necesidad de realizar pruebas moleculares para el diagnóstico de enfermedades causadas por Staphylococcus. A pesar de que se reporta el uso de cefoxitina para identificar SMR con una sensibilidad de $97.3 \%$ y una especificidad de $100 \%$ (Broekema et al., 2009), el CLSI recomienda la detección molecular del gene mecA como método más preciso para la predicción de la resistencia a la oxacilina (CLSI, 2012).

En esta investigación se identificó el gen mecA- 1 en cinco aislamientos de Staphylococcus obtenidos de las superficies intrahospitalarias. En las otras muestras analizadas de origen comunitario no se identificó este gen. La epidemiología de estas cepas con características de resistencia a antimicrobianos ha cambiado gradualmente desde su primer reporte, pasando de ser casos aislados y muy esporádicos a convertirse en uno de los organismos patógenos más prevalentes en superficies intrahospitalarias de humanos $\mathrm{y}$, por lo tanto, uno de los principales agentes causales de enfermedades nosocomiales (WHO, 2017). En clínicas veterinarias de pequeños animales de Estados Unidos se reportó una prevalencia de SAMR del 13.5\% en superficies en contacto con animales y humanos (Van Balen et al., 2013), sugiriendo la transmisión de estas bacterias de los humanos a los animales. No existe un consenso del tratamiento que se debe realizar a las superficies y del papel que estas desempeñan en la transmisión de patógenos (Dancer, 2009); sin embargo, algunos estudios han demostrado que las superficies contaminadas pueden actuar como fuente potencial de agentes asociados con infecciones nosocomiales (Donskey, 2013). En este estudio se evidenció el papel que desempeñan las superficies 
en el mantenimiento de este tipo de patógenos, el cual posiblemente es más importante que el que desempeñan las personas que están en continuo contacto con los hospitales veterinarios.

Se confirma que la mayoría de SMR presentan multirresistencia a otros antibióticos como aminoglucósidos, tetraciclina, trimetoprim/sulfametoxazol y macrólidoslincosamidas como eritromicina, tal y como lo reporta Salem et al. (2017). En forma similar, Calik et al. (2015), Othman et al. (2017) y Singh et al. (2017) encontraron diferentes tasas de resistencia de SMR frente a los antibióticos comúnmente empleados en la práctica clínica, como eritomicina $(50 \%)$, ciprofloxacina y trimetoprim/sulfametoxazol (22.3-40\%).

Los resultados de esta investigación también demostraron la utilidad de la PCR convencional y la secuenciación en la identificación de especies de Staphylococcus estrechamente relacionadas. El gen 16S rRNA codifica una región altamente conservada presente en todas las bacterias; sin embargo, pese a que se utiliza para la identificación de CNS (Clarridge, 2004), existen otros genes que pueden tener un mayor poder discriminatorio entre especies similares dentro del mismo género. Genes como tuf, el cual codifica para el factor de elongación $T u$ (constituyente esencial del genoma bacteriano), puede identificar de forma más precisa las especies altamente similares (Hwang, 2011). De esta forma, la PCR convencional y la secuenciación se postulan como herramientas diagnósticas que se pueden utilizar de manera alternativa a las metodologías convencionales y automatizadas.

En conclusión, los resultados de esta investigación proporcionan información acerca del uso de diferentes metodologías para la identificación y determinación de los perfiles de resistencia de Staphylococcus spp, validando la utilidad de las herramientas moleculares convencionales para identificar y caracterizar aislamientos de interés en sa- lud pública. Ha sido demostrado que el gen $m e c A$ no es el único responsable de proporcionar resistencia a la meticilina, por lo que se recomienda emplear otros genes reguladores pertenecientes al complejo mec (mecI y mecRI), los cuales también desempeñan un papel importante en el proceso de inducción de resistencia a los antimicrobianos (McKineey, 2001; Hung et al., 2011). En cuanto a las pruebas de fenotipo, el método de difusión de disco de cefoxitina se considera un mejor predictor que la oxacilina para la detección de la resistencia heterogénea a la meticilina (CLSI, 2016).

\section{Literatura Citada}

1. Acosta-Pérez G, Rodríguez-Abregón G, Longoria-Revilla E, Castro-Mussot $M E$. 2012. Evaluación de cuatro métodos para la detección de Staphylococcus aureus meticilino-resistente de muestras clínicas en un hospital regional. Salud Pública México 54: 1-6.

2. Aleixandre-Górriz I, DomínguezMárquez V, Martínez-Macías $O$, Colomina J, Guerrero A. 2014. Prevalencia de Staphylococcus aureus portadores del gen mecA sensibles a cefoxitina: OS-SARM. Rev Esp Quimioter 27: 215-216.

3. Broekema NM, Van Tam T, Monson TA, Marshall SA, Warshauer DM. 2009. Comparison of cefoxitin and oxacillin disk diffusion methods for detection of mecA-mediated resistance in staphylococcus aureus in a largescale study. J Clin Microbiol 47: 217-219. doi: 10.1128/JCM.01506-08

4. Calik Z, Karamese M, Acar O. 2015. Prevalence and antimicrobial-resistance of Staphylococcus aureus isolated from blood culture in University Hospital, Turkey. Glob J Infect Dis Clin Res 1: 10-13.

5. Chung M, Antignac A, Kim C, Tomasz A. 2008. Comparative study of the susceptibilities of major epidemic clones of methicillin-resistant Staphylococcus 
aureus to oxacillin and to the new broadspectrum cephalosporin ceftobiprole. Antimicrob Agents Ch 52: 2709-2717. doi: 10.1128/AAC.00266-08

6. Clarridge JE. 2004. Impact of $16 \mathrm{~S}$ rRNA gene sequence analysis for identification of bacteria on clinical microbiology and infectious diseases. Clin Microbiol Rev 17: 840-862. doi: 10.1128/ CMR.17.4.840-862.2004

7. [CLSI] Clinical and Laboratory Standards Institute. 2012. Performance standards for antimicrobial susceptibility testing, Approved Standard-1 $11^{\text {th }}$ Edition. CLSI document M02-A11. [Internet]. Available in: https://researchgate.net/file.PostFileLoader.html?id$=58139 \mathrm{aa} 4615 \mathrm{e} 27240754 \mathrm{da}-03 \&$ asse$\mathrm{tKey}=\mathrm{AS} \% 3 \mathrm{~A}-422233756704$ 774\%401477679780485

8. [CLSI] Clinical and Laboratory Standards Institute. 2016. Performance standards for antimicrobial susceptibility testing, Approved Standard-26 $6^{\text {th }}$ Edition. CLSI document M100S [Internet]. Available in: http://ljzx.cqrmhospital.com/upfiles/201601/ 20160112155335884.pdf

9. Cosseau C, Romano-Bertrand $S$, Duplan H, Lucas O, Ingrassia I, Pigasse C, Roques C, Jumas-Bilak E. 2016. Proteobacteria from the human skin microbiota: species-level diversity and hypotheses. One Health 2: 33-41. doi: 10.1016/j.onehlt.2016.02.002

10. Dancer SJ. 2009. The role of environmental cleaning in the control of hospital-acquired infection. J Hosp Infect 73: 378-385. doi:10.1016/j.jhin.-2009.03.030

11. Deurenberg $R H$, Vink $C$, Kalenic $S$, Friedrich $A W$, Bruggeman $C A$, Stobberingh EE. 2007. The molecular evolution of methicillin-resistant Staphylococcus aureus. Clin Microbiol 13: 222235. doi: 10.1111/j.1469-0691.2006.01573. $\mathrm{x}$

12. de Lencastre H, Oliveira D, Tomasz A. 2009. Antibiotic resistant Staphylococcus aureus: a paradigm of adaptive power. Curr Opin Microbiol 10: 428-435. doi: 10.1016/j.mib.2007.08.003.Antibiotic
13. Donskey CJ. 2013. Does improving surface cleaning and disinfection reduce health care-associated infections? Am J Infect Control 41(Suppl 5): 12-19. doi: 10.1016/j.ajic.2012.12.010

14. Fajardo M, Hidalgo R, Gaitán J, Sánchez-Silos R, Martín-Cordero P. 2016. Sobre los métodos microbiológicos para la detección de la resistencia a oxacilina en Staphylococcus coagulasa negativos. Rev Esp Quim 29: 244-248.

15. Foster TJ. 2017. Antibiotic resistance in Staphylococcus aureus. Current status and future prospects. FEMS Microbiol Rev 41: 430-449. doi: 10.1093/ femsre/fux007

16. Geha DJ, Uhl JR, Gustaferro CA, Persing DH. 1994. Multiplex PCR for identification of methicillin-resistant staphylococci in the clinical laboratory. $\mathrm{J}$ Clin Microbiol 32: 1768-1772.

17. Hanssen AM, Ericson JU. 2006. SCCmec in staphylococci: genes on the move. FEMS Immunol Med Mic 6: 820. doi: 10.1111/j.1574-695X.2005.00009.x

18. Heikinheimo A, Johler S, Karvonen L, Julmi J, Fredriksson-Ahomaa M, Stephan R. 2016. New dominant spa type 2741 in livestock-associated MRSA (CC398-MRSA-V) in Finnish fattening pigs at slaughter. Antimicrob Resist Infect Control 5: 6. doi: 10.1186/s13756016-0105-8

19. Hoet AE, Johnson A, Nava-Hoet RC, Bateman S, Hillier A, Dyce J, Gebreyes WA, et al. 2011. Environmental methicillin-resistant Staphylococcus aureus in a veterinary teaching hospital during a nonoutbreak period. Vector-Borne Zoonotic Dis 11: 609-615. doi: 10.1089/vbz.2010.0181

20. Hung KH, Yan JJ, Lu YC, Chen HM, Wu JJ. 2011. Evaluation of discrepancies between oxacillin and cefoxitin susceptibility in coagulase-negative staphylococci. Eur J Clin Microbiol 30: 785788. doi: 10.1007/s10096-011-1156-7 
21. Hwang SM, Kim MS, Park KU, Song J, Kim E-C. 2011. Tuf gene sequence analysis has greater discriminatory power than $16 \mathrm{~S}$ rRNA sequence analysis in identification of clinical isolates of coagulase-negative staphylococci. J Clin Microbiol 49: 4142-4149. doi: 10.1128/ JCM.05213-11

22. Ito T, Hiramatsu K, Tomasz A, De Lencastre H, Perreten, Holden MTG, Coleman DC, et al. 2012. Guidelines for reporting novel mecA gene homologues. Antimicrob Agents Chemoter 56: 4997-4999. doi: 10.1128/ AAC.01199-12

23. John MA, Burden JS, Stuart I, Reyes $R C$, Lannigan $R$, Milburn $S$, Diagre $D$, et al. 2009. Comparison of three phenotypic techniques for detection of methicillin resistance in Staphylococcus spp reveals a species-dependent performance. J Antimicrob Chemoter 63: 493496. doi: 10.1093/jac/dkn527

24. Johnson KN, Andreacchio $K$, Edelstein PH. 2014. Detection of methicillin-resistant coagulase-negative staphylococci by the vitek 2 system. J Clin Microbiol 52: 3196-3199. doi: 10.1128/JCM.01162-14

25. Mckinney TK, Sharma VK, Craig WA, Archer GL. 2001. Transcription of the gene mediating methicillin resistance in Staphylococcus aureus (mecA) is corepressed but not coinduced by cognate mecA and beta-lactamase regulators. J Bacteriol 183: 6862-6868. doi: 10.1128/JB.183.23.6862-6868.2001

26. Mendoza-Olazarán S, Morfín-Otero $R$, Villarreal-Treviño L, RodríguezNoriega E, Llaca-Díaz J, CamachoOrtiz A, González GM, et al. 2015. Antibiotic susceptibility of biofilm cells and molecular characterisation of Staphylococcus hominis isolates from blood. Plos One 10: e0144684. doi: 10.1371/journal.pone.0144684

27. Mujica E, Mujica A, Sánchez MP, Sánchez M, Sánchez A 2011. Presencia de los genes mec $A$ y blaZ como mecanismos de desarrollo de resistencia a â- lactámicos en cepas de Staphylo-coccus aureus. Rev Cient LUZ 1: 123-129.

28. Othman EW, Elsayed ZM, Farag KM. 2017. Genetic and phenotypic study of methicillin-resistant Staphylococcus aureus among patients and health care workers in Mansoura University Hospital, Egypt. Iran J Microbiol 9: 82-88.

29. Otto M. 2010. Staphylococcus colonization of the skin and antimicrobial peptides. Expert Rev Dermatol 5: 183195. doi: $10.1586 /$ edm. 10.6

30. Relman DA, Loutit JS, Schmidt TM, Falkow S, Tompkins LS. 1990. The agent of bacillary angiomatosis. New Engl J Med 323: 1573-1580.

31. Rose H, Hoar B, Kutz SJ, Morgan ER. 2014. Exploiting parallels between livestock and wildlife: predicting the impact of climate change on gastrointestinal nematodes in ruminants. Int $\mathrm{J}$ Parasitol 3: 209-219. doi: 10.1016/ j.ijppaw.2014.01.001

32. Safdar N, Narans L, Gordon B, Maki DG. 2013. Comparison of culture screening methods for detection of nasal carriage of methicillin-resistant Staphylococcus aureus: a prospective study comparing 32 methods. J Clin Microbiol 46: 3163-3166. doi: 10.1128/ JCM.41.7.3163-3166.2003

33. Schmidt T, Kock MM, Ehlers MM. 2017. Molecular characterization of Staphylococcus aureus isolated from bovine mastitis and close human contacts in South African dairy herds: genetic diversity and inter-species host transmission. Front Microbiol 8: 511. doi: 10.3389/fmicb.2017.00511

34. Singh S, Malhotra $R$, Grover $P$, Bansal R, Galhotra S, Kaur R, Jindal $N$. 2017. Antimicrobial resistance profile of methicillin-resistant Staphylococcus aureus colonizing the anterior nares of health-care workers and outpatients attending the remotely located tertiary care hospital of North India. J Lab Physicians 9: 317-321. doi: 10.4103/ JLP.JLP_8_17 
35. Song MD, Wachi M, Doi M, Ishino F, Matsuhashi M.1987. Evolution of an inducible penicillin-target protein in methicillin-resistant Staphylococcus aureus by gene fusion. FEBS Lett. 221: 167-171. doi: 10.1016/0014-5793(87)80373-3

36. Stapleton PD, Taylor PW. 2002. Methicillin resistance in Staphylococcus aureus: mechanisms and modulation. Sci Prog 85: 57-72. doi: 10.3184/003685002783238870

37. Tesch W, Strassle A, Berger-Bachi B, O'Hara D, Reynolds P, Kayser FH. 1988. Cloning and expression of methicillin resistance from Staphylococcus epidermidis in Staphylococcus carnosus. Antimicrob Agents Ch 32: 1494-1499. doi: 10.1128/AAC.32.10.1494

38. van Balen J, Kelley C, Nava-Hoet RC, Bateman S, Hillier A, Dyce J, Wittum TE, et al. 2013. Presence, distribution, and molecular epidemiology of methicillin-resistant Staphylococcus aureus in a small animal teaching hospital: a year-long active surveillance targeting dogs and their environment. Vector-Borne Zoonot 13: 299-311. doi: 10.1089/vbz.2012.1142

39. Van Duin D, Paterson DL. 2016. Multidrug-resistant bacteria in the community. Infect Dis Clin N Am 30: 377-390. doi: 10.1016/j.idc.2016.02.004
40. Watanabe K, Nakaminami H, Azuma C, Tanaka I, Nakase K, Matsunaga N, Okuyama K, et al. 2016. Methicillinresistant Staphylococcus epidermidis is part of the skin flora on the hands of both healthy individuals and hospital workers. Biol Pharm Bull 39: 1868-1875. doi: 10.1248/bpb.b16-00528

41. [WHO]. World Health Organization. 2014. Antimicrobial resistance. Global report on surveillance. [Internet]. Available in: http://apps.who.int/iris/ bitstream/handle/10665/112642/ 9789241564748 _eng.pdf; jsessionid=5E14F3-0E590D241C7C6BF56B29CF6085? sequence $=1$

42. [WHO] World Health Organization. 2017. Antimicrobial resistance [Internet]. Available from: http://www.who.int/ mediacentre/factsheets/fs194/

43. Zhang HZ, Hackbarth CJ, Chansky KM, Chambers HF. 2001. A proteolytic transmembrane signaling pathway and resistance to â-lactams in staphylococci. Science 291: 1962-1965.

44. Zuniga E, Melville PA, Saidenberg $A B S$, Laes MA, Gonsales FF, Salaberry SRS, Gregori F, et al. 2015. Occurrence of genes coding for MSCRAMM and biofilm-associated protein Bap in Staphylococcus spp isolated from bovine subclinical mastitis and relationship with somatic cell counts. Microb Pathogenesis 89: 1-6. doi: 10.1016/j.micpath.2015.08.014 